\title{
« Bilder ? - Wo das Urbild? » : F.H. Jacobi et la recherche platonicienne du modèle
}

«Bilder? - Wo das Urbild? »: Friedrich Heinrich Jacobi und die platonische Suche nach dem Urbild

"Bilder? - Wo das Urbild?": F.H. Jacobi and the platonic rechearch for the intelligible model

\section{Pierre Jean Brunel}

\section{OpenEdition}

\section{Journals}

Édition électronique

URL : http://journals.openedition.org/ceg/319

DOI : $10.4000 /$ ceg.319

ISSN : 2605-8359

\section{Éditeur}

Presses Universitaires de Provence

\section{Édition imprimée}

Date de publication : 5 avril 2017

Pagination : 49-70

ISBN : 979-10-320-0103-5

ISSN : 0751-4239

\section{Référence électronique}

Pierre Jean Brunel, « «Bilder? - Wo das Urbild? » : F.H. Jacobi et la recherche platonicienne du modèle », Cahiers d'Études Germaniques [En ligne], 72 | 2017, mis en ligne le 05 octobre 2018, consulté le 26 novembre 2020. URL : http://journals.openedition.org/ceg/319; DOI : https://doi.org/10.4000/ceg.319 


\title{
"Bilder? - Wo das Urbild?" \\ F.H. Jacobi et la recherche platonicienne du modèle
}

Pierre Jean BRUNEL

Université Lumière Lyon 2

\begin{abstract}
Poser dans toute son ampleur la question du modèle, de l'imitation et de la copie exige assurément de se tourner vers Platon. Friedrich Heinrich Jacobi en avait conscience parce qu'il avait une solide connaissance de l'histoire de la philosophie. Mais il ne suffit pas de rappeler cette perspective historique et philologique. Il importe surtout de comprendre pourquoi il ne semble précisément plus possible de comprendre Platon comme il se comprenait lui-même, en d'autres termes pourquoi la question platonicienne du modèle ou des Idées est devenue obscure, voire obsolète pour les Lumières. C'est Woldemar qui nous introduit le mieux à cette tension entre l'exténuation des anciennes idées platoniciennes et leur rémanence en soulignant la dimension existentielle des enjeux (philosophiques et religieux) liés à la crise de la raison. Kant avait remis en cause la possibilité de restituer le sens archétypal du modèle en s'appuyant sur une reformulation critique des Idées platoniciennes qui entendait affaiblir le prestige des Idées d'un point de vue théorique tout en préservant leur validité d'un point de vue pratique. Or, le modèle est désormais perçu dans ce roman comme " insondable ". Loin de régler le problème théologico-politique la solution kantienne semble exacerber les dissensions entre l'individu et le tout. Il nous faut partir de cette situation où s'exprime un mouvement inquiet de recherche.
\end{abstract}

Les écrits philosophiques de F.H. Jacobi permettent de préciser dans quelle mesure l'abstraction des images révèle le vide dès lors que l'idée platonicienne du Bien devient incompréhensible. À cet égard, le rapprochement avec le nominalisme hobbesien mérite une attention particulière puisque Hobbes critique les images et l'idolâtrie en se référant à Platon pour élaborer sa conception de l'Etat moderne. C'est l'analogie entre le modèle et le Verbe qui suggère une conception dynamique et ontologique renouvelée du modèle. La critique du criticisme kantien met en lumière l'importance de l'imagination dans la nouvelle philosophie idéaliste, notamment dans le projet fichtéen. Jacobi montre comment la philosophie subordonne le modèle à la copie. La question du schématisme kantien est le cœur du problème, le schème étant à ses yeux une " image sans image (!) ". Plus profondément il convient de mettre en évidence l'oubli du discours de l'être dans la philosophie qui apparente cette dernière à la sophistique. À suivre Jacobi, nous devons relire le Sophiste puisqu'il constitue 
la première théorie de l'image d'envergure. L'inconditionné recherché par la philosophie idéaliste correspond à ce que les platoniciens nommaient l'infıni. Mais Jacobi se demande si cet apeiron n'est pas une image trompeuse. Il convient donc de s'interroger sur la propension de la philosophie idéaliste à produire un phantasme alors même que cette dernière critique les images. L'allusion à la querelle des images dans Von den göttlichen Dingen und ihrer Offenbarung (cf. infra, n. 69) insiste enfin sur le sens aporétique de l'image et du modèle. La controverse entre iconodoules et iconoclastes repose en effet sur des concepts hérités du platonisme, notamment l'hypostase. Or, la pensée aussi bien littéraire que philosophique de Jacobi ne vise t-elle pas précisément à sauvegarder la notion de personne?

\section{Un modèle « insondable »? \\ La question du vide et des images dans Woldemar}

Dans le roman Woldemar (1796) le personnage éponyme s'interroge sur les images en recherchant éperdument le modèle ${ }^{1}$. On ne saurait trop insister sur le fait que ces termes ("Bilder " et "Urbild ") sont issus de la tradition platonicienne. "De pures ombres! Des figures fluentes, déconcertantes! Des images? Mais où trouver le type original ${ }^{2}$ ? ". Cette question revêt une grande portée pour saisir les enjeux de la philosophie contemporaine, car Jacobi joindra ce même extrait littéraire en appendice à sa Lettre à Fichte (1799) quelques années plus tard. Ces images représentent selon Woldemar les différentes " opinions sur le bonheur " (" Meynungen über Glückseligkeit ${ }^{3}$ ") qui divergent selon les personnes et les époques. Jacobi fait allusion au début du livre VII de La République où Platon évoque la situation des prisonniers dans la caverne qui ne perçoivent que " les ombres " (" sous l'effet d'un feu. L'anabase, la sortie du souterrain, force le prisonnier qu'on aurait détaché à voir les choses réelles telles qu'elles sont véritablement ${ }^{4}$. Mais il faut s'accoutumer progressivement à cette contemplation de la vérité, nous dit Socrate, il faut une éducation de l'âme pour entreprendre ce cheminement sans être ébloui. Le prisonnier verrait d'abord " les ombres qu'il distinguerait

1. F.H. Jacobi, Romane II. Woldemar, Texte, éd. par Carmen Götz et Walter Jaeschke, avec la collaboration de Dora Tsatoura. Werke, vol. 7, 1, Hamburg/ Stuttgart-Bad Cannstatt, Meiner/ frommann-holzboog, 2007, p. 269 [abréviation : Jacobi, Woldemar].

2. F.H. Jacobi, Lettre sur le nihilisme, et autres textes. Présentation, traduction et notes par Ives Radrizzani, Paris, Flammarion (coll. "GF »), 2009, p. 110 [abréviation: F.H. Jacobi, Lettre sur le nihilisme]; " Lauter Schatten! - Fließende, verwirrende Gestalten! ... Bilder? - Wo das Urbild? ", in F.H. Jacobi, Jacobi an Fichte, in Schriften zum transzendentalen Idealismus, vol. 2, 1, éd. par W. Jaeschke et Irmgard-Maria Piske, avec la collaboration de Catia Goretzki, Hamburg/ Stuttgart-Bad Cannstatt, Meiner/ frommann-holzboog, 2004, p. 256 [abréviation : Jacobi, Jacobi an Fichte].

3. Jacobi, Woldemar, p. 269.

4. Friedrich Heinrich Jacobi, "Einleitung in des Verfassers sämmtliche philosophische Schriften (1815) ", in Schriften zum transzendentalen Idealismus, vol. 2, 1, 2004, p. 407-408. 
plus facilement, et après cela, sur les eaux, les images des hommes et celles des autres réalités qui s'y reflètent, et plus tard encore ces réalités elles-mêmes ${ }^{5}$ ". L'expression " fließende, verwirrende Gestalten " se réfère précisément à ces figures " sur les eaux ", aux troublants reflets des réalités. Les images multiples ont une similitude avec le modèle, avec l'Idée ${ }^{6}$. En cherchant le " type original ${ }^{7}$ " Woldemar montre des dispositions pour la philosophie qui part des opinions confuses qui s'entrechoquent dans la cité pour connaître la vérité. Woldemar désire savoir ce que vise l'esprit humain lorsque l'homme aspire à la vertu, ce qu'il a " en vue ${ }^{8}$ " lorsqu'il dirige son regard vers cet " objet ". Conformément aux liens tissés en grec entre le verbe voir (idein) et l'idée (idea) Jacobi insiste sur cette visée eidétique de l'esprit, sur la perception des images qui ravive le souvenir du suprasensible. Or, cette connaissance ne semble plus possible, car le modèle est désormais " insondable ".

Ward das Urbild unerforschlich, wie konnte je die Einsicht des Guten zuverläßig werden? Wie konnte der Wille des Guten nur sich selbst erkennen, sich selbst verstehen, bey sich bleiben, und ein unveränderlicher Wille werden? [...] Mir graute, sagte Woldemar, vor der Finsterniß und Leere, die in mir und um mich entstand. ${ }^{9}$

Woldemar se réfère implicitement au livre VII de la République, le terme de " Einsicht " traduit cette idea du Bien que Socrate estime difficile à voir, et pourtant " c'est elle que doit voir celui qui veut agir de manière sensée, soit dans sa vie personnelle, soit dans la vie publique ${ }^{10}$ ". Tandis que l'idée platonicienne

5. Platon, La République, traduction de Pierre Pachet, Paris, Gallimard (coll. "Folio/essais "), 516a-b.

6. “'Eikon' désigne en grec une image qui présente un fort aspect de similitude, nous dirions aujourd'hui 'une icône', à la différence d'autres formes de représentations imitatives qui ne dévoilent pas la vérité du modèle; le même terme désigne aussi un reflet, c'est-à-dire l'image immédiatement réfléchie par un miroir, comme dans la Médée d'Euripide ou dans $L a$ République elle-même ", in Jean-François Mattéi, Platon et le miroir du mythe. De l'âge d'or à l'Atlantide, Paris, PUF (coll. "Thémis Philosophie "), 1996, p. 118.

7. C'est ainsi que Charles Vanderbourg traduit Urbild. Jacobi n'a pas jugé nécessaire de modifier cette traduction dans ce passage de Woldemar en français, cf. F.H. Jacobi, Lettre sur le nihilisme, p. 183.

8. "Was er [der menschliche Geist], indem er wahrhaft und allein auf diesen Gegenstand gerichtet sey, wahrhaft und allein im Auge habe? ", in Jacobi, Woldemar, p. 269.

9. Jacobi, Jacobi an Fichte, p. 256.

10. " [...] en assimilant la région qui apparaît grâce à la vue au séjour dans la prison, et la lumière du feu en elle à la puissance du soleil, et en rapportant la montée vers le haut et la contemplation des choses d'en-haut à la montée de l'âme vers le lieu intelligible, tu ne seras pas loin de ce que je vise, en tout cas, puisque c'est cela que tu désires entendre. Un dieu seul sait peut-être si cette visée se trouve correspondre à la vérité. Voilà donc comment m'apparaissent les choses: dans le connaissable, ce qui est au terme, c'est l'idée du bien, et on a du mal à la voir, mais une fois qu'on l'a vue on doit conclure que c'est elle, à coup sûr, qui est pour toutes choses la cause de tout ce qu'il y a de droit et de beau, elle qui dans le visible a donné naissance à la lumière et à celui qui en est le maître, elle qui dans l'intelligible, étant maîtresse elle-même, procure vérité et intelligence; et que c'est elle que doit voir celui qui veut agir de manière sensée, soit dans sa vie personnelle, soit dans la vie publique [nous soulignons] ", in Platon, La République, 517b-e. 
du Bien est certes " au-delà de l'essence ${ }^{11}$ ", mais oriente de manière décisive la vie pratique, Woldemar désespère de pouvoir appréhender le modèle, il sent peser sur lui l'influence de la philosophie contemporaine qui entreprend de réformer en profondeur la vie politique, sociale et religieuse en Europe. Jacobi pense sans nul doute à la doctrine kantienne qui établit que cette idée du Bien apparaissant " au terme " de l'analyse est inconnaissable, et rompt ainsi avec l'ascension vers la " contemplation de ce qui est ${ }^{12}$ ". Si la "volonté du bien " ne peut plus " se connaître et se comprendre " cela signifie un obscurcissement des fins visées naturellement par la pensée et l'action humaines. Woldemar est effrayé par ces " ténèbres " qui l'environnent, mais règnent aussi en lui. L'individu est affecté dans le tréfonds de son âme par cette disparition de l'intellection platonicienne, mais aussi son appartenance à la cité. Privé de la lumière du modèle, l'individu demeure prisonnier de la caverne de la cité, aussi éclairée soit-elle, puisqu'il doit se contenter de percevoir seulement des opinions fluctuantes et relatives. La sérénité et l'enjouement avec lesquels Socrate conversait avec ses amis sur le Bien ont disparu pour laisser place à la mélancolie ("Schwermuth "). L’inquiétude sur le lieu où se trouve le modèle trahit assurément le désir impétueux de ne pas demeurer prisonnier de la caverne et de se libérer de la convention, du nomos, à l'instar d'Hyperion dans le roman de Hölderlin ${ }^{13}$. En employant le mot " Einsicht " Jacobi renoue avec la définition de la sagesse telle que Socrate l'expose au début du livre VI de La République. Les " natures philosophes " sont " amoureuses du savoir capable de leur donner une vision de l'essence qui ne cesse d'être, sans errer sous l'effet de la naissance et de la corruption ${ }^{14}$ ". Jacobi dépeint ces errements consécutifs à la modification de la rationalité, la raison nouvelle s'épuisant dans sa propre réflexivité. Lorsque Socrate affirme que le gardien de la cité ne doit point être " aveugle ", mais avoir " la vue perçante ", il insiste sur l'importance d'avoir dans l'âme un " modèle clair ${ }^{15}$ ". Il importe d'être capable, " comme le feraient des peintres, de tourner les yeux vers ce qui est le plus vrai, de s'y reporter à chaque fois [...] ". Les images du peintre ne sont pas méprisées puisque la représentation est nécessaire, mais sans le modèle aucune clarté n'est possible. L'âme privée de modèle ne peut plus s'orienter lucidement. Cette expérience des images pose la question du nihilisme. Dès lors que la source ontologique et le modèle se sont dissipés, les Formes ou les Idées sont vides. Dénuée d'intellection, la pensée de l'âme qui s'adonne alors aux images et aux reflets ne risque t-elle pas d'être confinée à la réflexion?

\footnotetext{
11. Ibid., 509b.

12. Ibid., 518c.

13. Les références platoniciennes sont abondantes dès le Fragment. Cf. Pierre Jean Brunel, "La recherche de la vérité dans le Fragment von Hyperion de Hölderlin ", Le Texte et l'Idée, 2014/28, p. 57-74.

14. Platon, La République, 485 b.

15. Ibid., 484 c.
} 


\title{
«Bilder-Ideen und Wort-Welt ». Hobbes et l'herméneutique philosophique du monde
}

Selon Jacobi, la philosophie critique s'efforce de montrer que " le concept et la parole " ("Begriff und Wort ") créent une illusion ("Täuschung ") qu'il faut dissiper pour assainir le sol de la métaphysique. Or cette critique des Idées prive de sens l'existence en la laissant exsangue, dénuée de densité ontologique. Dans sa Lettre à Fichte, Jacobi présente cette interprétation de la nature de l'homme qui repose sur l'activité des images et des concepts.

\begin{abstract}
Oder war vielleicht dieser Wille nur die unmittelbare Folge des an allgemeine Begriffe und Bilder geknüpften persönlichen Bewußtseyns; nur der allen Naturen wesentliche Trieb der Selbsterhaltung in rein vernünftiger Gestalt? Dann hatte es keinen Gegenstand, als seine eigene Thätigkeit; und aller Tugenden Urbild und Quelle war die bloße rein - und leere Form des Daseyns im Gedanken: Persönlichkeit ohne Person und Personen - Unterschied. ${ }^{16}$
\end{abstract}

Ce que visait l'éducation platonicienne fait l'objet d'une explication scientifique qui renvoie le sujet à lui-même, à son activité immanente. Il ne subsiste plus qu'une conscience réflexive liée à des " concepts et images généraux ». Le résultat principal de la critique du modèle grec est une idéalisation grandissante et un rétrécissement du moi. La vie est devenue une image, une représentation, à tel point que l'individu perçoit sa propre vie comme une " ombre $^{17}$ ", cette copie d'une réalité qui se dérobe. Dans le débat entre le réalisme et l'idéalisme Jacobi insiste après Rousseau, et avec l'aide de Buffon, sur le " sentiment de l'existence ". D'autre part, Jacobi fait probablement allusion à Hobbes lorsqu'il parle de "l'instinct de conservation de soi ". Il importe de souligner pour notre propos l'importance que ce dernier accorde à " l'enchaînement, ou suite, des images $^{18}$ ". Hobbes considère ce qu'il appelle le " discours mental ${ }^{19}$ ". Il récuse surtout la scolastique des universités (qu'il nomme " aristotelity ${ }^{20}$ "), mais s'oppose aussi nettement aux Idées issues de la tradition platonicienne en se fondant sur un nominalisme afin de critiquer " les essences abstraites " et les "formes substantielles ${ }^{21}$ ". Les termes qui désignent l'être ne sont " nullement les noms des choses, mais des signes, [... ${ }^{22}$ ". La conception hobbesienne de la pensée suppose une défınition très simplifiée de la " compréhension ${ }^{23}$ " commune à l'homme et à l'animal. La compréhension propre à l'homme réside dans les

16. Jacobi, Jacobi an Fichte, p. 256.

17. Cf. " Hier ist der Fall, mit Homers Achill auszurufen: 'Lieber ein Bettler unter den Lebendigen, als ein König unter den Schatten!' ", in Jacobi, Woldemar, p. 268. Jacobi se réfère ici au rite d'invocation des morts, à la nekya du chant XI de L'Odyssée.

18. Thomas Hobbes, Léviathan (1651), chap. 3, traduction, introduction, notes et notices par G. Mairet, Paris, Gallimard, 2000, p. 85-94.

19. Ibid., p. 86.

20. Cf. le chapitre 46 " Des ténèbres produites par la vaine philosophie et les traditions fabuleuses ", in ibid., p. 906-936.

21. Ibid., p. 917.

22. Ibid., p. 919.

23. Ibid. 
pensées formées " par la série et la liaison des noms de choses [...] $]^{24}$ ". C'est précisément à cette activité immanente et individuelle que fait allusion Jacobi dans ses Lettres sur Spinoza.

\begin{abstract}
Wir eignen uns das Universum zu, indem wir es zerreissen, und eine unseren Fähigkeiten angemessene, der wirklichen ganz unähnliche Bilder-Ideen und Wort-Welt erschaffen. Was wir auf diese Weise erschaffen, verstehen wir, in so weit es unsere Schöpfung ist, vollkommen; was sich auf diese Weise nicht erschaffen läßt, verstehen wir nicht. ${ }^{25}$
\end{abstract}

Selon Jacobi l'homme ne peut comprendre qu'en créant un univers verbal, ou encore des images intellectuelles. Nous ne pouvons comprendre que ce que nous pouvons créer. Mais cette herméneutique des images et des idées détruit nécessairement l'unité ontologique afın que nous puissions nous approprier la réalité. Jacobi suggère ainsi qu'il est salutaire pour la philosophie elle-même de garder à l'esprit ce qui échappe à la compréhension scientifique du monde, ce dont nous ne pouvons pas nous faire d'image.

C'est au chapitre 45 (" De la démonologie ") du Léviathan que Hobbes traite du problème de l'image, du culte rendu aux images et de l'idolâtrie. Pour préciser sa définition des images il donne l'exemple du reflet (" shew ") d'une " chose dans l'eau, par réflexion ou réfraction, ou du soleil, des étoiles à la faveur d'une vision directe dans l'air ${ }^{26}$ ". Il s'agit selon nous d'une allusion à Platon qui lui permet de rapprocher les idées et les idoles, et d'établir " qu'il ne peut y avoir d'images d'une chose invisible ${ }^{27}$ ». Tandis que Hobbes conçoit en 1651 le projet de construire l'État moderne en dissipant les " ténèbres » et l'abstraction scolastique, le personnage de Woldemar constate à la fin du XVIII ${ }^{e}$ que la transformation de la raison entreprise par la philosophie moderne a aggravé cette abstraction en abandonnant la theoria: le modèle n'est plus une " source ", au sens ontologique du terme; il est devenu une généralité, un concept sans lien avec le particulier. Jacobi centre son analyse sur la personne parce qu'elle met en lumière les enjeux éthiques, religieux et politiques de cette grande transformation mise en branle par la philosophie. Le roman décrit la crise de la raison qui affecte la personne en se concentrant sur le personnage éponyme.

Tandis que Kant s'appuie sur une reformulation critique des Idées platoniciennes pour fonder sa réforme, Jacobi ne manquera pas de s'interroger sur ce renversement du modèle platonicien, et ainsi de confronter l'original platonicien à la copie revue et corrigée par Kant. Selon Jacobi, l'attitude de Kant envers le platonisme est ambiguë. Kant veut assainir et affermir le sol sur

24. Ibid., p. 84. Cf. " L'image qui est engendrée en l'homme (ou en toute autre créature douée de la faculté d'imaginer) par des mots ou par d'autres signes volontaires, est ce que nous appelons généralement compréhension [c'est Hobbes qui souligne]; et cela est commun aux hommes et aux bêtes " (ibid.).

25. F.H. Jacobi, Über die Lehre des Spinoza in Briefen an den Herrn Moses Mendelssohn (1785), Erweiterungen der zweiten Auflage (1789), Beylage VII, in Gesamtausgabe Werke. Schriften zum Spinozastreit, éd. par Klaus Hammacher und I. M. Piske, vol. 1, 1. Hamburg, Meiner frommann-holzboog, 1998, p. 249.

26. Hobbes, Leviathan, p. 888-889. Cf. aussi Platon, La République, 516 a-b.

27. Hobbes, Leviathan, p. 889. 
lequel s'édifie la métaphysique moderne en décrédibilisant la théorie des Idées. La confiance dans la possibilité de se tourner vers les Idées est ainsi fortement ébranlée, car celles-ci ne sont plus à proprement parler des archétypes du réel, mais sont devenues seulement régulatrices. Pour en dénoncer le caractère illusoire Kant souligne la dimension littéraire de ces Idées platoniciennes. Il n'est pas anodin que ce soit précisément la littérature qui dévoile alors la portée existentielle de ce bouleversement. Le roman se propose de mieux décrire ce que la philosophie entreprend de faire. Jacobi montre comment la raison peut être en proie à la plus grande confusion dès lors que la recherche du modèle archétypal paraît vaine.

\section{De l'original à la copie. La réforme kantienne des Idées platoniciennes et le modèle biblique}

Dans Über das Unternehmen des Kritizismus, die Vernunft zu Verstande zu bringen, und der Philosophie überhaupt eine neue Absicht zu geben (1802) Jacobi conteste vigoureusement la doctrine morale kantienne qui transforme la liberté en fantôme ("Gespenst ") et la divine Providence en "problème ". Jacobi estime que cette problématisation s'éloigne de l'expérience tout en prétendant lui rendre justice. Les modèles ("Urbilder ${ }^{28}$ ") montrent ce qui est incompréhensible, à savoir le miracle de la liberté. Les images originales du Bien et du Beau suscitent en l'homme un désir que le monde immanent ne saurait donner. Jacobi soutient que l'homme s'élève au-dessus de la nature et de la finitude grâce à ces archétypes qualifiés de divins. La moralité porte l'empreinte ("Abdruck ") de cette présence du divin dans la vie. Jacobi décrit cette relation érotique aux Idées d'origine platonicienne en marquant la distinction paulinienne entre l'esprit et la loi. "Voulais-tu connaître ta moralité à partir de la loi, le magnifıque modèle à partir de la copie réductrice? ${ }^{29}$ ". À suivre Jacobi, le modèle peut être compris comme analogue au Verbe, et ce serait une folie de s'imaginer qu'une " magnifique décision " (" herrlicher Entschluß ") puisse être prise en suivant seulement la loi. On sent poindre dans cette polémique contre Kant l'inquiétude que la nouvelle morale ne contribue à rapetisser les actions humaines en enserrant " la vertu et la liberté " dans " les rets rationnels de la morale ${ }^{30}$ ". Il importe de souligner que cette défense du modèle ne correspond pas dans l'esprit de Jacobi à un idéal qu'il faudrait ensuite mettre en œuvre. C'est originairement dans l'action que se révèlent la présence et la prégnance du modèle qui demeure fondamentalement dynamique, tandis que les maximes ou les postulats kantiens semblent n'être

28. F.H. Jacobi, Über das Unternehmen des Kritizismus, die Vernunft zu Verstande zu bringen, und der Philosophie überhaupt eine neue Absicht zu geben (1802), in Schriften zum transzendentalen Idealismus, p. 329 [abréviation: Über das Unternehmen des Kritizismus].

29. "Wolltest du aus dem Gesetze deine Sittlichkeit erkennen, aus verkleinernder Copie das herrliche Urbild? ", in Jacobi, Über das Unternehmen des Kritizismus, p. 329.

30. " [...] ehe man sie [Tugend und Freyheit] mit dem vernünftigen Netze der Moral umstrickte: [...] " (ibid., p. 329). 
qu'une copie, autrement dit incapables d'impulser l'action parce qu'ils sont coupés de la vie ${ }^{31}$. Jacobi fonde sa conception très singulière de la révélation sur la relation vivante entre la copie et le modèle qu'il distingue de l'idole. Il se réfère aussi bien à Confucius, à Socrate qu'au Christ pour comprendre l'action vertueuse des " grands hommes".

Nach Gottes Bilde geschaffen, Gott in uns und über uns; Urbild und Abbild, getrennt und doch in unzertrennlicher Verbindung: das ist die Kunde, die wir von ihm haben, und die einzig mögliche; [...]. Weisheit, Gerechtigkeit, Wohlwollen, freye Liebe, sind keine Bilder, sondern Kräfte, von denen man die Vorstellung nur im Gebrauch selbsthandelnd erwirbt. ${ }^{32}$

L'homme se distingue du modèle divin, mais il est à son image; le modèle et la copie sont donc séparés, mais aussi étroitement unis. Selon Jacobi, c'est en agissant que le sujet donne naissance au modèle divin présent en lui, avant même d'avoir reçu un enseignement conceptuel sur le modèle. Jacobi insiste sur cette dimension pratique et intérieure pour contester la possibilité d'une amélioration morale de l'homme par l'enseignement des idées ("Unterricht ${ }^{33}$ ") si elles sont conçues abstraitement comme des idéaux, ou pour employer un langage qui nous est aujourd'hui familier, comme des valeurs. C'est parce que la vertu ou le bien existent " en soi " que nous pouvons en faire l'expérience. La réforme de Kant a exténué l'intelligibilité des Idées platoniciennes. Il importe donc à Jacobi de revenir au modèle pour savoir que l'on a " plus qu'une idée ${ }^{34}$ "lorsque l'on se tourne vers l'idée du Bien ${ }^{35}$. Jacobi se réfère à Platon pour indiquer que la sortie de la caverne suppose de voir le Bien en soi, c'est-à-dire l'être qui échappe au devenir, mais dont nous retrouvons la copie dans le monde immanent (" diesseits abgebildet »). La bonté du modèle intelligible est comparable, selon Jacobi, à la lumière du soleil dans le livre VI et VII de la République. Sans cet " au-delà "

31. «Moralität ist Abdruck dieses Göttlichen im Leben, nicht Wirkung einer kalten, leeren Maxime [...]. Unsterblichkeit beruht nicht auf einem müssigen Postulat; wir fühlen sie in unserm freyen Handeln und Wirken " (ibid.). Cf. les remarques lumineuses de Jan Patočka sur " la manière de surmonter l'abstraction, le déchirement, l'appauvrissement de la vie ", in "L'évolution philosophique et esthétique de Hegel ", in L'art et le temps, traduit par E. Abrams, Paris, Presses Pocket, 1990, p. 194.

32. F.H. Jacobi, "Von den göttlichen Dingen und ihrer Offenbarung " (1811), in Schriften zum Streit um die göttlichen Dinge und ihre Offenbarung, vol. 3, éd. par Walter Jaeschke, Hamburg, Stuttgart-Bad Cannstatt, Meiner, frommann-holzboog, 2000, p. 42 [abréviation: Jacobi, Von den göttlichen Dingen und ihrer Offenbarung (1811)].

33. Jacobi, ibid.

34. "Und so bedarf es nur der Idee, um zu wissen, daß man hier mehr als Idee, daß man Wirklichkeit und Wahrheit überschwänglich habe " (ibid., p. 45). Le terme " überschwänglich " est connoté négativement par Kant, et évoque l'usage extravagant et enthousiaste des Idées, surtout dans le domaine théologique. Cf. Yves-Jean Harder: "Kant et la question de la transcendance ", in Métaphysique et philosophie transcendantale selon Kant, textes réunis et publiés par Mai Lequan, Paris, L’Harmattan, 2005, p. 79-102, et particulièrement p. 98-99 où Harder rapproche le sens négatif du terme " transcendant " de " überfliegend " et de " überschwenglich ".

35. "Nur wer jenseits, über dem Entstehenden und Vergehenden, das an sich Wahre, Gute und Schöne erblickt hat, findet es auch diesseits abgebildet wieder [...] ", in Jacobi, Von den göttlichen Dingen und ihrer Offenbarung, p. 135-136. Cf. aussi Platon, La République, 507 b-c, 508 d-e, 517d, et 519a. 
(" jenseits ") aucune chose ne pourrait apparaître, ni naître. Le modèle dispense la vie au monde sensible et visible. Or la philosophie contemporaine rompt avec cette conception du Bien. Dans sa discussion avec Fichte, Jacobi insiste sur le legs kantien pour dégager le sens plasmateur de la production des images. En considérant l'imagination dans le système kantien, Jacobi entend saisir comment la vie devient une représentation schématique, et tente d'affaiblir par ricochet la doctrine fichtéenne.

\section{L'imagination comme «tisseuse ». La critique de l'idéalisme et le phantasme de l'apeiron}

Dans Über das Unternehmen des Kritizismus, die Vernunft zu Verstande zu bringen, und der Philosophie überhaupt eine neue Absicht zu geben (1802), Jacobi se réfère principalement à la Kritik der reinen Vernunft (1781, 1787). La faculté de juger transcendantale, et en particulier bien sûr le schématisme de l'entendement pur éveillent son intérêt ${ }^{36}$. Sa comparaison de l'imagination avec une tisserande n'est pas sans faire songer à l'image ("Gleichniß ") du bas tricoté utilisée dans la Lettre à Fichte ${ }^{37}$. De Kant à l'idéalisme de Fichte, l'imagination est devenue un processus d'individuation dont le pouvoir n'est pas seulement créateur, mais aussi destructeur. Au moyen de la science les objets sont décomposés, et donc progressivement déconstruits par des concepts toujours plus généraux. Cette compréhension scientifique immanente permet ensuite de reproduire les objets à l'aide de l'imagination qui est au fond une alma mater:

Bildlich könnte man die Einbildungskraft, diese alma mater, als eine Weberin darstellen. Das ursprüngliche reine Bewußtseyn wäre die Kette oder der Aufzug am Gewebe, die sinnlichen Empfindungen der Einschlag. Mit den Füßen brächte die Wirkerin Handlung in die Kette (,intellektuelle, ganz unfigürliche Synthesis'); mit den Händen triebe sie den Einschlag durch (,figürliche productive Synthesis'). ${ }^{38}$

Nous remarquons que Jacobi, à l'instar de Socrate, a recours aux images dans l'exposition des doctrines. Cette question des images et de la philosophie était au centre du débat entre Jacobi et Herder au sujet de Lessing et Spinoza ${ }^{39}$. Sous l'influence d'une certaine interprétation de Spinoza le théologien Herder veut rejeter les images au nom de la rigueur philosophique. Il soutient que les images n'ont pas de sens philosophique. Herder nous rappelle que l'art d'écrire ésotérique/ exotérique était familier à Lessing et à Leibniz, et que les images ne seraient

36. Sur l'image, voir en particulier le premier chapitre de la doctrine transcendantale de la faculté de juger, "Von dem Schematismus der reinen Verstandesbegriffe ", in I. Kant, $K r V$, B 179-181/ A $140-142$.

37. Jacobi, Brief an Fichte, in Schriften zum transzendentalen Idealismus, p. 203.

38. Jacobi, Über das Unternehmen des Kritizismus, p. 280. Cf. I. Kant, KrV, B 150-152. Jacobi se réfère ici au §24, "Von der Anwendung der Kategorien auf Gegenstände der Sinne überhaupt ".

39. Cf. notre article, "Le dévoilement de l'existence et les images. Philosophie, théologie et poésie. Herder, Jacobi et la querelle du panthéisme ", Le Texte et l'Idée, Nancy, 2012/26, p. 1-26. 
pour eux qu'un habillage ${ }^{40}$. Il importe selon Herder d'éviter la " catachrèse d'une image $^{41}$ ", ainsi que les " anthropopathies " et un " anthropomorphisme " qui serait même " souvent presque continu " dans l'œuvre de Leibniz par exemple ${ }^{42}$. Jacobi et Herder mettent en garde contre l'image trompeuse, mais Jacobi estime qu'il serait plus raisonnable d'analyser la source scripturaire de l'image pour mesurer combien la philosophie s'égare en modifiant en profondeur la raison. En ramenant la raison à la raison (c'est-à-dire à l'entendement philosophant, selon le programme fixé par la nouvelle philosophie idéaliste: Über das Unternehmen des Kritizismus, die Vernunft zu Verstande zu bringen, und der Philosophie überhaupt eine neue Absicht zu geben, 1802), la philosophie moderne veut éliminer ce qui semble être pure folie. Mais, en niant les résonances bibliques obvies dans l'interprétation de l'être et de l'image, la philosophie confère précisément à l'entendement un pouvoir déraisonnable. Selon Jacobi, l'homme ne peut faire autrement que d'anthropomorphiser sa représentation de Dieu, car il est créé selon Sa ressemblance ${ }^{43}$. L'homme est à l'image de Celui qui existe en soi-même. Le mot " Gleichniß " qui signifıe ici image ou comparaison fait écho à ce qui est égal (" gleich "), c'est-à-dire ce qui est le même, l'être stable. Dans la relation de l'homme à Dieu, nous ne pouvons faire abstraction de la forme divine que Dieu donne à l'homme, cette image ressemblante explique pourquoi l'homme est nécessairement obligé de donner une forme humaine à Dieu. C'est la raison qui fait que l'homme est un homme, c'est-à-dire " l'image de Dieu " (" Ebenbild Gottes ${ }^{44}$ "). Du " théomorphisme " à l'anthropomorphisme, c'est le mystère de la raison et du logos dont Jacobi décrit les inchoations dans Von den göttlichen Dingen und ihrer Offenbarung (1811) ${ }^{45}$. Cette position incline Jacobi à reconsidérer la dimension rhétorique de la philosophie, les relations entre les mots et les choses, ou encore entre la forme et la chose (Gestalt/Sache).

Jacobi a conscience que la conjonction de la rhétorique et de la philosophie heurte le systématisme philosophique contemporain. L'image rhétorique permet de révéler l'ambition inouïe de la philosophie qui modifie la raison pour produire " l'idée de l'inconditionné ", " une représentation entièrement vide, mais qui n'en demeure pas moins le principe de la raison, la matrice de tous

40. "Also wenn wir von Gott reden, lieber keine Bilder! Auch in der Philosophie ist dies unser erstes Gebot, wie im Gesetz Moses ", in Herder, Gott. Einige Gespräche, in Schriften zu Philosophie, Literatur, Kunst und Altertum (1774-1787), vol. 4, éd. par J. Brummack und M. Bollacher, Frankfurt am Main, Deutscher Klassiker Verlag, 1994, p. 759 [abréviation: Herder, Gott].

41. Herder, Gott, p. 760.

42. " [...] daher denn die Anthropopathien, ja oft beinah ein fortgesetzter Anthropomorphismus ", in Herder, Gott, p. 730.

43. "Geschaffen nach Seinem Bilde, ein Gleichniß Seiner, des in sich Seyenden [c'est Jacobi qui souligne] ", in Jacobi, Von den göttlichen Dingen und ihrer Offenbarung (1811), p. 112. Cf. Genesis, 1. Mose, I, 26-27.

44. Jacobi, Ibid.

45. F.H. Jacobi: « Den Menschen erschaffend theomorphisirte Gott. Nothwendig anthropomorphisirt darum der Mensch ", in Jacobi, Von den göttlichen Dingen und ihrer Offenbarung (1811), p. 112. 
ses concepts ${ }^{46}$ ». L'entendement métamorphose l'idée de l'inconditionné en quelque chose de subjectif, ce qui est réel devient alors un simple idéal qui, selon Jacobi, sera ensuite objectivé ${ }^{47}$. L'entendement, qui occupe une place éminente dans la philosophie nouvelle, produit une " image trompeuse, un fantôme de l'inconditionné 48 ". Jacobi ajoute que cette image est une idole (" Götze 49 "). " La philosophie du néant absolu ${ }^{50}$ " est une idolâtrie. En voulant lutter contre la foi du charbonnier la philosophie adore une idole de l'absolu que Jacobi décrit comme un concept plus vaste et général qui tend idéalement à embrasser la totalité. À suivre Jacobi, l'inconditionné visé par la philosophie moderne est quelque chose de complètement indéterminé (" das durchaus Unbestimmte "), et correspond à ce que Platon et les pythagoriciens nommaient l'infini ${ }^{51}$. Selon nous, Jacobi traduit ici en allemand le terme grec apeiron qui désigne ce qui est illimité et ne possède aucune forme ${ }^{52}$. Ce dieu métaphysique est selon Jacobi un " phantasme " ("Phantasm "), c'est-à-dire un simulacre. C'est une image illusoire ("Wahnbild ") de ce qui ne peut exister. Ce qui est infıni est informe et exprime une image contradictoire du non-être. La philosophie moderne a l'illusion de pouvoir donner naissance à ce dieu métaphysique, mais révèle en fait qu'elle n'a pas suffisamment médité la dialectique entre l'être et le non-être héritée de Platon et des présocratiques.

\section{Le schème kantien comme " image sans image (!) » et la subordination du modèle à la copie depuis Aristote}

Le schème permet la transition entre les concepts purs de l'entendement et les phénomènes ${ }^{53}$. Néanmoins selon Jacobi, le schème est " une image purement produite par l'entendement, mais qui n'est pas autorisée à s'appeler ainsi parce qu'elle ne doit pas être empirique, mais une image générale sans image $\left(!^{54}\right.$ ".

46. " eine durch und durch leere Vorstellung, aber nichts desto weniger das Princip der Vernunft, die Gebärmutter aller ihrer Begriffe ", in Jacobi, Über das Unternehmen des Kritizismus, p. 282.

47. Jacobi, Von den göttlichen Dingen und ihrer Offenbarung (1811), p. 110.

48. "Ein Trugbild, ein Gespenst des Unbedingten, tritt an des wahrhaft Unbedingten Stelle; [...] ", in Jacobi, Von den göttlichen Dingen und ihrer Offenbarung (1811), p. 111.

49. "Dieses Trugbild, dies Gespenst, - dieser Götze, [...] " (ibid.).

50. " die Philosophie des absoluten Nichts " (ibid., p. 110).

51. "Das leere Chaos, die Ungestalt, das durchaus Unbestimmte (Platon und die Pythagoräer nannten es das Unendliche) ist das Unbedingte, das Absolute des Verstandes; jenes Phantasm, [...]" (ibid. p. 110).

52. Cf. Platon, Philèbe et F.H. Jacobi: « Nennet Gott nicht das unendliche Wesen, saget Platon; denn dem unendlichen widerstehet das Daseyn; es ist wesentlich wesenlos; [...] ein seyendes Nichtseyn. Sein Bild ist das Wahnbild der Ungestalt [nous soulignons], [...] ", in Jacobi, Von den göttlichen Dingen und ihrer Offenbarung (1811), p. 15.

53. Cf. Raphaël Ehrsam, "Le rôle du schématisme dans la connaissance: une interprétation sémiotique ", in Sophie Grapotte, Mai Lequan, Marguit Ruffing (dir.), Kant et la science: la théorie critique et transcendantale de la connaissance, Paris, Vrin, 2011, p. 145-154.

54. " ein von dem Verstande rein hervorgebrachtes Bild, das aber, weil es kein empirisches, sondern ein allgemeines unbildliches Bild (!) seyn soll, den Namen nicht tragen darf ", in 
La synthèse est à la fois figurée et entièrement non figurée. En hypostasiant la sensibilité, Kant tente d'obtenir une image qui soit licite pour l'idéalisme critique ${ }^{55}$. Jacobi insiste sur le fait que l'union de la sensibilité et de l'entendement dans la doctrine kantienne met l'homme en contradiction avec lui-même, car l'une exige ce qui est déterminé et " un maximum de contenu ", tandis que l'autre exige " le maximum d'étendue, de général ${ }^{56}$ ". La morale et la religion portent la marque de cette maximisation déchirante. Nous pouvons noter que, selon Jacobi, le schématisme de l'entendement " anthropomorphise ", c'est-à-dire " incarne ${ }^{57}$ " l'existence d'un être infini que la raison postule. Cependant, comme cette " incarnation " contredit le postulat d'un être infini, la possibilité et l'existence de cet être demeurent "problématiques ${ }^{58}$ ".

Jacobi explique, à l'aide de Kant, comment l'individu devient le jouet de cette imagination qui est " une pure affabulation vide " (" ein reines leeres Dichten ${ }^{59}$ "). Le terme de "Einbildung " indiquant bien qu'il s'agit d'une illusion, voire d'une tromperie ("Betrug "). Kant nous apprend que dieu, la liberté et l'immortalité ne sont plus des objets d'une intuition possible par les sens, mais seulement des concepts de la raison, des Idées passées par l'étamine critique. Il est impossible, selon Jacobi, de revenir à des représentations objectivement vraies et réelles, et d'avoir une sincère " confiance " ("Vertrauen ") dans ces Idées tellement nous sommes maintenant " trop bien informé " ("zu gut unterrichtet ") de leur origine et de leur nature intrinsèque. Nous ne pouvons plus " oublier que ces Idées ne sont que des "fictions heuristiques, des foci imaginarii', [... ${ }^{60}$ ". Dans ce contexte, un des passages les plus importants commenté par Jacobi est Die Disziplin der reinen Vernunft in Ansehung der Hypothesen (I, 3) dans la Transzendentale Methodenlehre à la fin de la Kritik der reinen Vernunft.

Die Vernunftbegriffe sind, wie gesagt, bloße Ideen, und haben freilich keinen Gegenstand in irgend einer Erfahrung, aber bezeichnen darum doch nicht gedichtete und zugleich dabei für möglich angenommene Gegenstände [c'est Jacobi qui souligne]. Sie sind bloß problematisch gedacht, um, in Beziehung auf sie (als heuristische Fiktionen), regulative Prinzipien des Systematischen Verstandesgebrauchs im Felde der Erfahrung zu gründen. ${ }^{61}$

Les concepts de la raison sont de "simples Idées ", ce qui ne veut pas dire qu'elles désignent des objets fictifs, selon Kant. Le point le plus intéressant qui mérite d'être souligné, selon Jacobi, consiste à préciser que ces objets sont impossibles

Jacobi, Über das Unternehmen des Kritizismus, p. 280 (note 2).

55. Jacobi, Über das Unternehmen des Kritizismus, p. 319.

56. " ein Maximum des Inhalts ", " das Maximun des Umfangs, des Allgemeinen ", in Jacobi, Über das Unternehmen des Kritizismus, p. 281.

57. " er [der Schematismus des Verstandes] ist es also, der anthropomorphisirt, (Rel. inn. der Gr. d. bl. Vern. S. 82) verkörpert [...] ", in Jacobi, Über das Unternehmen des Kritizismus, p. 281.

58. " problematisch" (ibid.).

59. Ibid., p. 282.

60. Cf. ibid., p. 284 : " [...] vergessen, daß jene Ideen nur 'hevristische Fictionen, foci imaginarii' [...] sind ". Cf. aussi I. Kant, KrV, B 672/ A 644 ("Anhang zur transzendentalen Dialektik. Von den regulativen Gebrauch der Ideen der reinen Vernunft ").

61. I. Kant, $K r V$, B 799/ A 771. Il se réfère aussi à KrV, B 392/ A 335 ("System der transzendentalen Ideen "), in Jacobi, Über das Unternehmen des Kritizismus, p. 285. 
puisqu'ils ne sont pas fictifs ${ }^{62}$. Le lecteur peu attentif est soulagé d'apprendre que ces simples Idées ne sont cependant pas des fictions, mais il lui échappe peut-être que Kant ajoute aussitôt que ces objets ne peuvent pas être tenus pour possibles. Il faudrait alors comprendre qu'il n'est pas possible que de telles Idées soient pensables. Avec beaucoup de précaution, mais aussi avec netteté, Kant affirme qu'il n'y a non seulement rien de véritablement objectif pour l'homme, mais il est impossible qu'il en soit autrement. Coupé " de tout ce qui est vrai et qui subsiste en soi " (" von allem Wahren, in sich Subsistirenden ") le sujet se représente toutes sortes de choses, mais il ne voit ainsi que " de manière aveugle " (" blindlings ${ }^{63}$ "). Le changement de l'âme (" Gemüthveränderung ${ }^{64}$ ") ne s'effectue que par la sensibilité qui détermine la subjectivité. Jacobi résume ainsi la situation: "Elle [la partie supérieure de l'âme] ne comprend qu'avec des concepts ce que l'autre partie ne voit pas; elle est aveugle avec des yeux voyants, tout comme l'autre partie est voyante avec des yeux aveugles ${ }^{65} \mathrm{n}$.

Dans l'importante introduction à ses œuvres (Einleitung in des Verfassers sämmtliche philosophische Schriften, 1815) Jacobi estime que la question du modèle et de la copie est essentielle pour clarifier les enjeux philosophiques et scientifiques.

\begin{abstract}
Es war seit Aristoteles ein zunehmendes Bestreben [...] das Urbild dem Abbilde, das Wesen dem Worte, die Vernunft dem Verstande unterzuordnen, ja in diesem jene ganz untergehen und verschwinden zu lassen. Nichts sollte fortan mehr für wahr gelten, als was sich beweisen, zweymal weisen ließe: wechselweise in der Anschauung und im Begriffe, in der Sache und in ihrem Bilde oder Worte; und in diesem nur, dem Worte, sollte wahrhaft die Sache liegen und wirklich zu erkennen seyn. ${ }^{66}$
\end{abstract}

Il est si difficile de se tourner vers le modèle à cause de cette subordination qui depuis Aristote donne la préséance à la copie. Cette conception qui fait autorité tend à " faire totalement sombrer et disparaitre " le modèle dans la copie, autrement dit à rendre impossible le cheminement platonicien vers la connaissance que Jacobi appelle de ses vœux. C'est en subordonnant la faculté de perception à la faculté de réflexion que la philosophie accomplit cette transformation. L'abstraction va de pair avec le développement des images parce qu'elle repose sur une activité adossée à la connaissance médiate. La raison est conçue à tort comme un processus réflexif qui élève les connaissances sensibles au niveau suprasensible, et dont l'effet spéculaire et spéculatif inverse ainsi le bas et le haut. Jacobi s'accorde avec la critique kantienne qui récuse cette connaissance de l'intelligible. La validité objective de ces Idées obtenues par négation reste indémontrable. Kant

62. Jacobi se réfère à I. Kant, $K r V$, B 271.

63. Jacobi, Über das Unternehmen des Kritizismus, p. 286. Cf. I. Kant, KrV, B 800/ A 772.

64. Ibid.

65. «Dieser Gemüthstheil begreift nur mit Begriffen, was der andere nicht sieht; er ist mit sehenden Augen blind, wie der andere mit blinden Augen sehend ", in Jacobi, Über das Unternehmen des Kritizismus, p. 286.

66. F.H. Jacobi, " Einleitung in des Verfassers sämmtliche philosophische Schriften ", in Schriften zum transzendentalen Idealismus, p. 379 [abréviation: Einleitung in des Verfassers sämmtliche philosophische Schriften]. 
est ici un recours pour montrer la fausseté de ce rationalisme qui se propose d'appréhender le vrai en soi à partir des connaissances sensibles. Jacobi s'appuie aussi sur Fries (Neue Kritik der Vernunft, $1807^{67}$ ) qui estime qu'Aristote a séparé les formes de la réflexion du reste de la connaissance matérielle. Il dénonce avec Fries l'usage de la méthode logique dans la philosophie leibnizo-wolffienne. Il importe donc de recouvrer le sens de la connaissance immédiate, autrement dit de la réceptivité du logos, en exhumant la raison oubliée ou détrônée au profit la réflexion. Le rationalisme des Lumières repose sur une confusion, c'est un rationalisme nominal (" Nominal-Rationalismus ${ }^{68}$ "). La philosophie organise de manière systématique un renversement entre le modèle et la copie qui sera gros de l'avenir, car les images et les paroles contiennent désormais ce qui est vrai.

\section{Le modèle et la recherche platonicienne de I'Un. L'inversion sophistique du modèle et de la copie}

Dans l'annexe C ("Beylage C ") de Von den göttlichen Dingen und ihrer Offenbarung (1811) Jacobi rappelle le sens original des Idées dans la doctrine platonicienne afin de saisir la nouveauté de l'interprétation kantienne. Les êtres réels particuliers ont pour cause ("Ursache ") les espèces, qui elles-mêmes ont pour cause les genres. Ainsi, les espèces et les genres platoniciens ne sont " pas des êtres simplement logiques ou nominaux ${ }^{69}$ ". Kant croit au contraire que ces espèces et ces genres sont la conséquence d'une "illusion logique " ", des concepts simplement déduits dont toute la vérité serait tirée de réalités qui leur préexistent. Jacobi compare les Idées de Platon (les genres) à l'idée d'un premier inventeur pour insister sur le fait que les Idées sont antérieures aux choses particulières.

[...], wie der Gedanke eines ersten Erfinders und das von ihm, nach diesem Gedanken, erschaffene Musterbild früher sind, als die Unendlichkeit der Nachbildungen nach der im Musterbilde offenbarten Absicht und Regel; dergestalt, daß diese spätere Vielheit erst möglich wurde durch jenes frühere Eine, und aus ihm hervorging. ${ }^{71}$

C'est l'Un qui précède le divers, le modèle est antérieur à ses copies, à ses reproductions infınies ${ }^{72}$. L'unité du modèle manifeste, ou révèle une intention et une règle qui rendent possibles les diverses copies. La doctrine platonicienne permet à Jacobi de clarifier le rapport de ses contemporains à la rationalité

67. Cf. Christian Bonnet, L'Autre École de Iéna. Critique, métaphysique et psychologie chez Jacob Friedrich Fries, Paris, Classiques Garnier, 2013.

68. Jacobi, Einleitung in des Verfassers sämmtliche philosophische Schriften, p. 383.

69. "[...] keine blos logische oder Nominalwesen, [...] ", in Jacobi, Von den göttlichen Dingen und ihrer Offenbarung (1811), p. 133.

70. " logische Täuschung " (ibid.).

71. Jacobi, Von den göttlichen Dingen und ihrer Offenbarung, p. 133-134.

72. "das Eine selbst [to hen auto] " (ibid, p. 133). Jacobi fait ici sûrement allusion à la réplique suivante de l'Étranger dans le Sophiste: "Mais dans ces conditions, n'est-il pas impossible que la chose soit l'un même [nous soulignons] ", in Platon, Sophiste, 245a-c. 
qui confondent l'unité obtenue par l'entendement ("Verstand ") et celle par la raison ("Vernunft ${ }^{73}$ "). L'unité de l'entendement à partir de la pluralité et $d u$ multiple est produite par " le concept et le mot " (" Begriff und Wort "). C'est pourquoi la confusion entre la raison comme faculté de perception immédiate et l'entendement ou la réflexion favorise immanquablement les images, c'est-à-dire aussi les mots au détriment du Réel. La cause première ne suppose pas une relation " simplement logique " mais ontologique. Si l'on ne s'interroge pas sur la manière dont est produite rationnellement l'unité, les résultats obtenus par la philosophie ont beau être prometteurs, ils n'en demeurent pas moins trompeurs. Le rationalisme est divisé, et surtout incapable de voir que sa cohérence repose sur une contradiction irrésolue qui agit comme une vis a tergo.

[...] dem trete nothwendig überall das Wort vor das Wesen; das Abbild, und des Abbildes trügerischer Schein vor das Urbild, so daß dieses, das Wahre und Wesenhafte, das nur sich selbst Gleiche, gar nicht mehr von ihm gesehen werde, und er vorgebe, gleich den Zauberern, auch sich selbst überrede, alles mit Worten machen zu können: in Wahrheit aber verliere ein solcher mit dem Grunde der Rede die Rede [c'est Jacobi qui souligne] selbst; denn was er vorbringe, sey leerer Schall - Trug und Lüge. ${ }^{74}$

La recherche de la vérité suppose la recherche de l'essence. Lorsque l'on pose simplement la question socratique " qu'est-ce que (le beau, le bien) ...? ", on vise une entité qui reste égale à elle-même, qui ne change pas. Jacobi se réfère ici au Cratyle ${ }^{75}$. Nous avons déjà eu l'occasion de commenter les références à ce dialogue platonicien dans l'annexe C, ainsi qu'au Sophiste et au Théétète ${ }^{76}$. Mais c'est le Timée de Platon qui éclaire singulièrement le problème de l'image

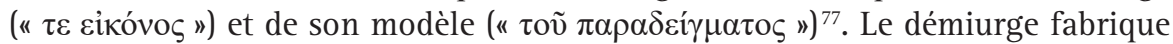
le monde en prenant pour modèle, pour paradigme ( $\pi \alpha \rho \alpha \delta \varepsilon^{\prime} \gamma \mu \alpha \tau \iota^{78}$ ") ce qui

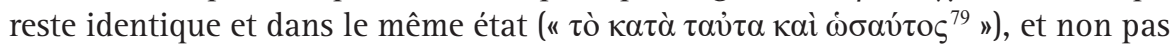
quelque chose qui est engendré (" $\tau$ ò $\gamma \varepsilon \gamma o v o ́ \varsigma^{80}$ "). La beauté du monde et la bonté du démiurge sont liées à la nature de cette pensée, de cette raison du fabricant que Timée qualifie de " meilleures des causes ${ }^{81}$ ". C'est probablement à ce passage du Timée que Jacobi se réfère lorsqu'il aborde la question du " culte des images " ("Bilderdienst ${ }^{82}$ "), nous y reviendrons ${ }^{83}$.

73. L'unité obtenue par la raison platonicienne est "l'un lui-même ": " das Eine selbst [to hen auto] ", in Jacobi, Von den göttlichen Dingen und ihrer Offenbarung, p. 133.

74. Ibid.

75. Platon, Cratyle, 439a-d.

76. Cf. Pierre Jean Brunel, Les Lumières platoniciennes de Friedrich Heinrich Jacobi, Paris, Pups, 2014, p. 277-294, en particulier la section " L'image et le phénomène ", p. 274-276.

77. Platon, Timée, 29b, présentation et traduction par Luc Brisson, Paris, Flammarion, 1999 [1992].

78. Platon, Timée, 28a.

79. Ibid., 29a.

80. Ibid., 28b.

81. Ibid., 29a.

82. Jacobi, Von den göttlichen Dingen und ihrer Offenbarung (1811), p. 47.

83. "'Gott ist' - sagt erhaben Timäus - 'was überall das Bessere hervorbringt:' Der Geist und die Gewalt des Guten. " (ibid., p. 46). 
Mais dans ces conditions, notre monde doit de toute nécessité être l'image de quelque chose. Il est bien sûr de la plus haute importance de commencer par le commencement naturel. Voici donc, concernant une image et son modèle, la distinction qu'il faut établir, étant admis que tout discours porte sur quelque chose et que ce sur quoi porte ce discours lui est apparenté. D’un coté donc, tout discours qui porte sur ce qui demeure, sur ce qui est stable et translucide pour l'intellect, cela ne doit en rien manquer d'être stable et inébranlable, pour autant qu'il est possible et qu'il convient à un discours d'être irréfutable et invincible; d'un autre coté, tout discours qui porte sur ce qui est la copie de ce dont on vient de parler, parce qu'il s'agit d'une copie, entretient avec la première espèce de discours un rapport d'image à modèle [nous soulignons]. Ce que l'être est au devenir, la vérité l'est à la croyance ${ }^{84}$.

Il faut distinguer deux sortes de modèle ou de paradigme. L'un regarde l'éternel en cherchant à reproduire "la forme [idea] et les propriétés [dunamis] " du modèle, tandis que l'autre regarde le devenir. L'idéalisme renonce en fait à cette idea originelle et promeut le second modèle qui se rapporte à la copie (" Eikóvos

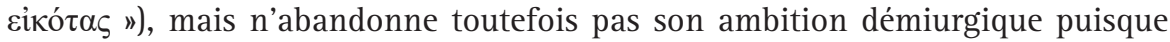
cette doctrine s'imagine imiter encore Platon tout en réformant sa théorie des Formes ${ }^{85}$. L'ordre ontologique qui régit les rapports entre l'image et le modèle est également épistémologique. La philosophie se détourne de la véritable science en privant le discours de son fondement ontologique. Lorsque Jacobi parle du " fondement du discours ", c'est de l'être dont il est question. Le Sophiste porte sur l'être et poursuit la discussion du Théétète, c'est pourquoi Jacobi se réfère expressément à ces dialogues ${ }^{86}$.

Dans le Sophiste, l'Étranger remarque que l'imitation est une espèce de production bien qu'elle ne produise " que des images, et non des réalités véritables ${ }^{87}$ ". Il divise en deux parties l'art de produire, l'une étant divine, l'autre humaine. La pensée créatrice, la science divine sont distinguées de la production humaine. Chacun de ces genres sera lui-même divisé, selon la méthode dialectique, pour faire la différence entre les choses réelles et les simulacres (phantasmata) ou les copies (eikones). L'art divin a aussi ses simulacres, comme les songes, les ombres et les reflets. Jacobi fait allusion à l'effet de miroir dans Platon qui permet d'illustrer la compréhension moderne de la rationalité, à savoir la réflexion qui " fait sur nos sens l'effet inverse de notre vue ordinaire ${ }^{88}$ ".

Dans la préface à ses œuvres philosophiques (Vorrede, zugleich Einleitung in des Verfassers sämmtliche philosophische Schriften, 1815) Jacobi renvoie à l'annexe C de Von den göttlichen Dingen und ihrer Offenbarung (1811) ${ }^{89}$. Il insiste sur le fait que le discours ("Rede ") doit être au centre des préoccupations puisqu'il est très difficile de se rendre compte de l'art du sophiste qui emploie des images pour établir la vérité de sa doctrine de l'unité du Tout (" Lehre der All-Einheit »), notamment celle de Schelling. Le discours est à l'image de la

84. Platon, Timée, 29b-c.

85. Erwin Panofsky, Idea. Ein Beitrag zur Begriffsgeschichte der älteren Kunsttheorie, Berlin/ Leipzig, Teubner, 1924.

86. Brunel, Les Lumières platoniciennes de Friedrich Heinrich Jacobi, p. 276.

87. Platon, Sophiste, 265a-265c.

88. Ibid., 266a-c. La description de l'inversion est encore plus détaillée in Platon, Timée, 46a-c.

89. Jacobi, Von den göttlichen Dingen und ihrer Offenbarung, p. 408. Voir aussi dans ce contexte la référence au Théétète, 152d-e (ibid., p. 406). 
doctrine: il s'écoule, car dans cette pensée du devenir tout est en perpétuel mouvement. Le logos se dégrade en logorrhée, car on ne peut dire ce qui est, la substance devient évanescente faute de substantifs ("Haupt- oder Nennworte ${ }^{90}$ "). Lorsque Jacobi compare cette philosophie à celle des magiciens il fait allusion à l'" art fantasmagorique " du " faiseur d'images ${ }^{91}$ " dont parle l'Étranger à Théétète dans le Sophiste. Les questions de l'un et du tout, de la parole et de la vérité que soulèvent les conceptions idéalistes contemporaines sont confrontées par Jacobi à la discussion de la thèse de Parménide sur l'être et le non-être. C'est dans le Sophiste que l'on trouve en effet la première théorie de l'image d'une telle ampleur dans la tradition philosophique ${ }^{92}$. L'" art de fabriquer des images " comprend deux genres, l'un qui copie, l'autre qui fait des " simulacres" ". $\mathrm{Au}$ moment de la dialectique descendante Platon montre que les images du sophiste sont trompeuses dans la mesure où elles se distinguent malaisément du modèle, contrairement à celles employées par le philosophe, comme celle de la caverne dans la République. L'inversion de la copie et du modèle empêche de " voir " le vrai, et nous abuse en présentant l'apparence comme étant l'essence. La dialectique se dégrade en sophistique. Le charlatan imite les réalités en retournant les mots contre son interlocuteur. Le sophiste propose une " copie " (" cíkós ») qui " reproduit l'original ${ }^{94}$ ". Il importe de relever que, selon Jacobi, la prédominance des images et des mots nous fait finalement perdre non seulement le " fondement du discours ", mais " le discours lui-même ". L'Étranger déclare dans le Sophiste qu'on pourrait penser que celui qui entreprend de dire le nonêtre ne dit rien, mais on peut aller plus loin, et affirmer que ce dernier " ne parle même pas ${ }^{95}$ ". L'art du sophiste est un " art de tromperie ${ }^{96}$ ", il trompe les gens " par des fantômes ". On retrouve ici les termes avec lesquels Jacobi fourbit sa critique du criticisme. Mais il faudra réfuter la thèse de Parménide, accomplir le parricide, pour aborder les problèmes des images, des imitations, des apparences et des discours faux ${ }^{97}$.

Perdre de vue le bon modèle, c'est vider la parole de sa substance. C'est le logos lui-même qui devient flatus vocis puisque les mots n'ont plus de fondement ontologique. Il ne subsiste rien dans le flux du discours qui ne vise que le devenir.

90. Jacobi, Einleitung in des Verfassers sämmtliche philosophische Schriften, p. 407.

91. Platon, Sophiste, 239c-240a.

92. Stanley Rosen, Plato's Sophist. The drama of original and image, New Haven, Londres, Yale University Press, 1983. Cf. Villela-Petit, "La question de l'image artistique dans le Sophiste ", in Pierre Aubenque, Michel Narcy (dir.), Études sur le Sophiste de Platon, Naples, Bibliopolis, 1991, p. 53-90.

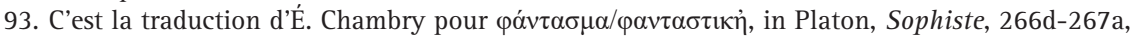
Paris, Flammarion (coll. "GF »), 1969, p. 141. Voir aussi, Sophiste, 236c. On peut aussi traduire ce " phantasme " par le terme d'illusion.

94. Platon, Sophiste, 235a-236c.

95. Ibid., 237e.

96. Ibid., 240c-240e.

97. « [...] mettre à la question la thèse de notre père Parménide [...] " (ibid., 241b-e). Cf. Monique Dixsaut, Platon et la question de la pensée. Études platoniciennes, vol. 1, Paris, Vrin, 2000, en particulier "Les deux espèces de productions d'images: vrais et faux semblants ", p. 295-298. 
Mais au moment où cet affaiblissement s'effectue, Jacobi constate une confiance démesurée dans la puissance de la parole qui prétend produire, faire advenir quelque chose de nouveau en imitant le pouvoir plasmateur. Lorsque Jacobi parle du magicien qui " se persuade lui-même être capable de tout produire avec des paroles ", on reconnaît le sophiste qui, selon Platon, " [...] se fait fort de tout créer par un seul art $[\ldots]^{98}$ ". Le sophiste est comme un peintre qui nous présente des " images parlées ${ }^{99}$ ". Dès lors, la philosophie platonicienne est un recours pour renouer avec le "discours lui-même ", avec la véritable dialectique qui procède par divisions (diairesis) comme l'étranger invite Théétète à le faire, et rappelle à la fin du dialogue la différence entre l'ignorance et la connaissance. La copie est liée au " paraître " (doxa), à l'opinion, cette imitation de la vérité. Parménide soutenait qu'il est impossible de prouver que le non-être existe. Or, l'Étranger dit que le non-être existe bel et bien, " autrement le faux ne pourrait pas être ${ }^{100}$ ". Jacobi dénonce la fausseté de ce rationalisme qui repose sur une funeste confusion entre le fondement et la cause dans la recherche de l'Un et de l'inconditionné ${ }^{101}$. Il est important de noter que, selon Jacobi, ce sophiste se trompe lui-même. Il est probable que Jacobi pense à celui qui imite ce dont il n'a pas connaissance. À la fin du Sophiste, l'Étranger fait une distinction entre l'imitation qui se fonde sur l'opinion, et celle qui se fonde sur la science. Faute de nom pour les désigner, il forge les expressions suivantes: la doxomimétique et l'imitation savante. Les noms font défaut pour nommer ce phénomène. Nombreux sont ceux qui ne connaissent pas "la vertu en général ", mais qui s'en forment une opinion, et imitent ce qu'ils prennent pour la vertu " dans leurs actes et dans leurs paroles ${ }^{102}$ ".

\section{Philosophie et religion: la querelle aporétique des images et l'hypostase}

Si l'on prend conscience du fait que la relation au modèle lui-même puisse disparaître, la situation du personnage Woldemar dans le roman éponyme devient moins énigmatique, car il ne dispose pour ainsi dire plus que d'une seule science, la doxomimétique qui, comme le précise M. Dixsaut, " redouble l'être apparent d'une apparence de connaissance ${ }^{103}$ ". Le modèle platonicien permet ainsi à Jacobi de critiquer la propension de la philosophie critique et idéaliste à l'abstraction, ainsi que l'illusion des images, tout en ne rejetant pas la raison et la

98. Platon, Sophiste, 234b.

99. Ibid., 234c-235a.

100. Ibid., 237a.

101. Birgit Sandkaulen, Grund und Ursache. Die Vernunftkritik Jacobis, München, W. Fink, 2000.

102. Platon, Sophiste, 267a-c.

103. Dixsaut, Platon et la question de la pensée, p. 300. "La doxomimétique a ceci de spécifique qu'imitateur, instrument, image produite mais aussi modèle, tout se confond dans un même acte de produire. Le modèle n'a plus aucune consistance, puisque la distinction entre l'extérieur et l'intérieur s'abolit. [...] Cette sorte d'imitateur produit, dans l'indiscernabilité du dehors et du dedans, le simulacre du paradigme qu'il prétend imiter " (ibid., p. 301). 
recherche philosophique quand cette dernière a recours aux images rhétoriques. Les rapports complexes entre la philosophie moderne et la religion sont au cœur des tensions entre le modèle et la copie.

La discussion sur la personne du Christ dans Von den göttlichen Dingen und ihrer Offenbarung permet à Jacobi de mettre en lumière les difficultés de la réforme kantienne. Ce dernier cite Der Wandsbecker Bote de Matthias Claudius pour comprendre l'" image " ("Bild ${ }^{104}$ ") du Christ telle que la comprend un véritable homme de foi. Claudius remarque que nous serions malavisés de nous moquer, et qu'il ne s'agit nullement d'une " simple idée " (" bloße Idee ${ }^{105}$ "). Cette allusion à Kant est l'occasion pour Jacobi de confronter cette position authentiquement religieuse à celle d'un philosophe. Ce dernier estime que ce qui est représenté "sous l'image et avec le nom du Christ " (" unter dem Bilde und mit dem Namen Christus ") peut inciter à mener une vie divine ou vertueuse. Il n'y pas d'" idolâtrie " ("Götzendienst ") tant que l'homme pieux vénère seulement " ce qui est divin en soi " (" was göttlich ist an sich "). L'essence se confond ici avec l'espace privé, et traduit une subjectivation de l'image de la personne du Christ. Le philosophe précise en effet qu'il est indifférent de savoir si cette représentation existe à l'extérieur, si ce concept de la personne du Christ correspond à la réalité, car seule "la vérité essentielle " (" die wesentliche Wahrheit ") importe. Le vocabulaire utilisé est clairement platonicien puisqu'il est question de s'élever sur les ailes des Idées ${ }^{106}$. Il faut garantir l'innocuité des idées religieuses en les essentialisant. Il sera ainsi possible d'errer et de se tromper sans péril. Dès lors que le modèle divin, en l'occurrence la personne du Christ, est exprimé par l'Idée et intériorisé, le philosophe ne s'alarme plus si l'homme pieux accorde plus d'importance à l'" habillement " de l'Idée, à ce qui est inessentiel plutôt qu'à l'essence du divin lui-même. La vraie religion, selon le philosophe platonicien et kantien, est celle de l'esprit. Il pense ainsi rejoindre pleinement les aspirations de l'homme religieux, même si ce dernier peut succomber à " une sorte de matérialisme religieux " (" eine Art von religiösen Materialismus "), c'est-à-dire " faire naître la chose de sa forme " (" die Sache aus ihrer Gestalt enspringen lassen ${ }^{107}$ »). Le philosophe se méfie et rejette le culte de l'image matérielle (Gestalt) au profit de l'esprit, du modèle idéal. Mais l'homme religieux refuse ce compromis philosophique qui tend à rendre la religion idéale et invisible. Jacobi se réfère à la " célèbre querelle sur les droits des images " (" bey dem berühmten Streite über die Rechte der Bilder ") des VIII et IXe siècles. Selon Jacobi, cette querelle théologique et politique est susceptible d'une application plus large, car sa portée intellectuelle n'est pas seulement historique. Jacobi formule la question suivante qui faisait broncher les théologiens : doit-on

104. "Welch in Bild! ", in Jacobi, Von den göttlichen Dingen und ihrer Offenbarung, p. 44.

105. Matthias Claudius, Der Wandsbecker Bote, Frankfurt am Main, Insel Verlag, 1975, p. 354.

106. " Du ersiehst durch ihn die Gottheit, so weit du sie ersehen kannst, indem du dich zu den höchsten Ideen mit ihm empor schwingst, und unschädlich irrend, wähnest, dich nur an ihm dazu empor zu schwingen [c'est Jacobi qui souligne] " (ibid.). Cf. Kant, KrV A 591/ B 619, et surtout $K r V$ B 658/ A 630, ainsi que Platon, Phèdre, 249e.

107. Jacobi, Von den göttlichen Dingen und ihrer Offenbarung, p. 44. 
vénérer et adorer aussi bien la divinité, l'essence divine du Christ que sa " forme corporelle " (" leibliche Gestalt ${ }^{108}$ ")? La réponse consista à affirmer que les deux dimensions sont unies indissociablement dans la " personne " du Christ. Le philosophe interprète cette réponse comme une victoire du culte des images (" der Bilderdienst ") et comme la naissance de l'orthodoxie. Jacobi fait allusion à la victoire théologique de l'iconophilie. Un concile fut en effet convoqué le 11 mars 843 , le jour où fut célébré pour la première fois la fête désormais annuelle du rétablissement de l'orthodoxie. Le messager de Wandsbeck ne peut accepter ce culte des images si le philosophe fixe comme condition que le vrai ne réside que dans l'idée. On peut alors reprocher à la philosophie moderne de succomber à un " chimérisme religieux ". Jacobi dévoile alors aussi bien la dimension religieuse et nihiliste de la philosophie qui a seulement le modèle à l'idée, que la dimension matérialiste de la religion qui accorde une grande importance à l'image visible et au corps. Tandis que la philosophie révèle que le culte religieux des images tend à l'idolâtrie, la religion révèle que l'iconoclasme philosophique produit un phantasme religieux.

Leicht ist aus der Sache, welche wir zu schlichten wünschten, nicht zu kommen. Auf den Vorwurf von religiösem Materialismus, sich selbst verborgenem Bilder - oder Götzendienst, den wir dem Boten machen, giebt er uns den Vorwurf von religiösem Chimärismus, Phantasterey, Selbstgötterey, Nihilismus zurück; [...].

La discussion sur les droits des images demeure aporétique, car aucune des deux parties ne peut trouver de solution satisfaisante. D'une part, la religion ne peut devenir entièrement invisible et intérieure comme le voudrait la philosophie, d'autre part la philosophie ne peut renoncer à se défaire des images et de leurs copies sans courir le risque de renoncer à elle-même. Cette question de l'iconoclasme et de l'idolâtrie permet à Jacobi de rappeler les tensions entre la matière et l'esprit, le sensible et l'intelligible, le visible et l'invisible. Il choisit délibérément de ne pas trancher ce débat. Le silence et la folie ironique $d u$ messager qui refuse à la fin de serrer la main du philosophe témoignent de l'impossibilité de régler la question entre la sobriété d'Athènes et la folie de Jérusalem. Dans ce passage de Von den göttlichen Dingen und ihrer Offenbarung Jacobi fait probablement allusion à Jean Damascène, l'auteur de la première synthèse théologique de l'icône qui rappelle que nous avons besoin des choses corporelles comme intermédiaires pour nous conduire aux choses intelligibles.

A. Besançon résume ainsi le propos de cet iconodoule dans L'Image interdite: " On voit que pour le Damascène l'image est hiérarchisée selon le degré de participation, plus ou moins intense et complète, au prototype ${ }^{109}$. "

Jacobi savait en effet qu'on ne peut formuler dans toute son ampleur la question d'origine platonicienne du prototype (Urbild) et de l'image (Bild) sans s'interroger sur la personne et l'incarnation, autrement dit sur l'hypostase. Birgit Sandkaulen parle à juste titre de " philosophie de la personnalité " (" Philosophie

108. Ibid., p. 47.

109. Alain Besançon, L'Image interdite, Paris, Gallimard (coll. " Folio/essais "), 1994, p. 239. 
der Personalität ${ }^{110}$ ») en la distinguant de la philosophie de la subjectivité des Modernes. Jacobi s'oppose à cette volonté de détruire la personnalité qui apparaît par exemple dans la philosophie de Schelling, la personnalité devant s'effacer devant la "sphère absolue de l'être ". Or, Jacobi ne suggère-t-il pas qu'il est illusoire de s'affranchir du degré de participation au prototype? Jacobi redoute qu'en visant l'absolu et en critiquant le paradigme du dieu personnel au nom de la liberté, l'iconoclasme philosophique n'asservisse l'homme à son phantasme.

110. Birgit Sandkaulen, " Dass, was oder wer? Jacobi im Diskurs über Personen ", in Walter Jaeschke, Birgit Sandkaulen (dir.), Friedrich Heinrich Jacobi. Ein Wendepunkt der geistigen Bildung der Zeit, Studien zum achtzehnten Jahrhundert, vol. 29, Hamburg, Meiner, 2004, p. 234. 
